\title{
Facile crystallization and proton conductivity of $\left[\mathrm{Zn}_{3}(\mathrm{BTC})_{2}\left(\mathrm{H}_{2} \mathrm{O}\right)_{12}\right]_{\mathbf{n}}$
}

\author{
M. Obulichetty and D. Saravanabharathi ${ }^{\mathrm{b}}$ \\ $\left\{\right.$ moc.apsc@psgtech.ac.in ${ }^{\mathrm{a}}$, apsc@psgtech.ac.in $\left.{ }^{\mathrm{b}}\right\}$ \\ ${ }^{a}$ Department of Applied Science,PSG College of Technology, Coimbatore - 641 004, \\ India., ${ }^{b}$ Department of Chemistry, PSG College of Technology, Coimbatore - 641 004, \\ India.
}

\begin{abstract}
This work describes the facile and one-step crystallization strategy for the preparation of the title complex through simple mixing of the aqueous reactants, and thus eliminates the need for hydrothermal synthesis. The structural study on the harvested crystalline material, confirms the absolute reproducibility of the title complex by this newer method as well. The suitability of our method in achieving the bulk purity of the title compound, is fully ascertained by elemental analysis, and by powder XRD methods. Due to the presence of metal bound water, and extensive hydrogen bonds in the structure, the system is considered to be very suitable for the proton conduction applications. However, despite the popularity of the compound in the field of MOFs, such studies were never performed. The compound's AC impedance experiments proves the proton conducting characteristics of the title complex under 100\% RH .The Ea value implies that the Grotthuss process is followed. Proton conductivity vanishes under dry conditions, but is restored upon re-exposing the sample to the humidity. As a result, the current study emphasises the utility of this well-known and non-hazardous framework for a variety of proton electrolyte applications.
\end{abstract}

Keywords: XRD, crystalline, MOFs, Proton conductivity.

\section{Introduction}

Metal-organic frameworks (MOFs) have gainedsubstantial interest in terms of design and synthesis due to their intriguing structural architectures and also because of their potential applications, ranging from gas separation, catalysis, optical phenomena, electric and magnetic materials, sensorsetc[1-4]. The MOFs have also been researched as proton-conductive electrolytes for applications in proton exchange membrane based fuel cells [5]. The MOFs, that possess the Lewis acidic charge carriers, and networks of hydrogen bonded arrays of water molecules are being explored as proton conducting electrolytes [1-6]. MOFs based proton electrolytes, are being reported in recent times [1-7].The presence of water molecules and humidity are the required attributes in establishing the $\mathrm{H}^{+}$conducting routes in the material. Such materials emerge as potential solid polymer electrolytes for a variety of applications, including fuel cells, humidity sensors, and hydrogen separators [1- 7].

Although the research on modern generation electrolytesare drawing interest [8], it is also essential to explore the known compounds for the purpose,that are already having the required structural features for proton conductivity [9]. In this connection, it is interesting to pick out 
the relevance of the title complex, which is an inexpensive and eco-friendly material, under the present scope. Its structural characteristics, as revealed by crystallographic reports [10, 11], are highly relevant for proton conduction applications, due to the cross-linked network of coordination assemblies, Coordinated water molecules are Lewis acidic, and the hydrogen bondscould transport the $\mathrm{H}^{+}$. However, despite the popularity of the title compound, it was never explored in this line so far, and thus falls in the scope of the present work.

In addition, the present work also explores a facile crystallization strategy for the title compound and enables the researchers to harvest bulk quantities of the compound, in an accelerated manner, under normal laboratory conditions itself, just by simply mixing the aqueous reactants.

\section{Experimental}

\subsection{General}

All chemicals and solvents were acquired from standard chemical vendors and utilised without any further purification.

\subsection{Physical measurements}

PXRD studies were performed inthe Shimadzu Lab Model 6000 XRD analyzer. Single crystal structure determination by X-ray diffraction was performed on a Bruker Axs Kappa Apex 2 CCD Diffractometer. The structure was solved by direct method with SHELXS-97 and refined using SHELXL-97 [12].

For conductivity measurements, powdered material was compacted into a pellet ofa 1.3 $\mathrm{cm}$ diameter with a thickness of $0.105 \mathrm{~cm}$ pellet under a pressure of $100 \mathrm{~kg} / \mathrm{cm}^{2}$.BioLogic SP$200(1 \mathrm{MHz}-10 \mathrm{mHz})$ was used for AC impedance under full closed humidity as before [9]. The $\mathrm{C}, \mathrm{H}$, analyses were performed in the ElementarVario EL III analyzers.

\subsection{Synthesis of $\left[\mathrm{Zn}_{3}(\mathrm{BTC})_{2}\left(\mathrm{H}_{2} \mathrm{O}\right)_{12}\right]_{\mathbf{n}}$}

Zinc acetate-dihydrate $(1 \mathrm{mmol}, 0.220 \mathrm{~g})$ was dissolved in $150 \mathrm{~mL}$ of distilled water to form a clear solution, into which a solution of 1,3,5 benzene tricarboxylic acid ( $1 \mathrm{mmol}, 0.210$ $\mathrm{g}$ in $50 \mathrm{~mL}$ of methanol) was progressively added and the resulting clear solution was preserved at room temperatures (open air).Within three hours, formations of X-ray quality fine crystals have been noticed at the bottom of the beaker, and bulk yields were obtained after 2 days.Colorless X-ray level crystals obtained were washed with distilled water, filtered and dried. Yield: $0.48 \mathrm{~g} \mathrm{(} \sim 5 \%$ with respect to zinc acetate-dihydrate). Elemental analysis Anal. [Calculated for $\mathrm{C}_{18} \mathrm{H}_{30} \mathrm{O}_{24} \mathrm{Zn}_{3}$ is $\mathrm{C}=26.16 \%, \mathrm{H}=3.73 \%$; Found: $\mathrm{C}=26.09 \%, \mathrm{H}=3.36$ $\%]$.

\section{Results and Discussion}

\subsection{Crystallization of $\left[\mathrm{Zn}_{3}(\mathrm{BTC})_{2}\left(\mathrm{H}_{2} \mathrm{O}\right)_{12}\right]_{n}$}

Yaghi et.al have harvested the $\left[\mathrm{Zn}_{3}(\mathrm{BTC})_{2}\left(\mathrm{H}_{2} \mathrm{O}\right)_{12}\right]_{\mathrm{n}}$ complex as single crystals, using the hydrothermal procedures, and have reported the structure of the iso-structural cobalt complex [10]. The molecular structure of the complex $[10,11]$ is composed of two dimensional zig-zag chains. It consists of two symmetry-in equivalent tetra aqua $\mathrm{Zn}$ (II) units and BTC ligands. The two carboxylate units of BTC ligand coordinate in a unidentate mode in the axial positions of a near perfect octahedral and symmetry-equivalent zinc centres, whereas the 
third carboxylate group of the BTCforms bidentate coordination to the equatorial positions of third, distorted, octahedral M(II) centre. Two oxygen atoms of water molecules coordinated with symmetry-equivalent $\mathrm{M}$ (II) centres are involved in hydrogen bonding with uncoordinated oxygen of BTC. Similarly the other two oxygen atoms of coordinated water molecules present in the third M(II) centre also forms hydrogen bonding with the two oxygen atoms of the water molecules present in the symmetry equivalent $\mathrm{M}(\mathrm{II})$ centres, thus generating a three dimensional network $[10,11]$. As mentioned in the introduction, all these structural attributes of the title complex add merit to the title complex to consider as proton conducting electrolyte, and thus require a thorough investigation in these line [9]. However, such electrochemical investigations always calls for a quicker and a facile synthetic route for the material [9], and thus become as an additional goal of the present work.

A room temperature, aqueous crystallization route for this material was unintentionally developed by Krishnamurthy et.al., during their exploration to characterize organic bases containing Zn-BTC complexes [11]. While using 4-aminopyridine as an organic base, they have obtained a white precipitate first, which after filtering, the filtrate yielded the crystalline $\left[\mathrm{Zn}_{3}(\mathrm{BTC})_{2}\left(\mathrm{H}_{2} \mathrm{O}\right)_{12}\right]_{\mathrm{n}}$ complex. The X-Ray structural features were found to be identical with that of Yagi et. al [10].

In this work, we reasoned that the stronger acidic BTC system would displace the acetate moiety from the zinc acetate precursor as acetic acid, and thus it might be possible to harvest the title complex just by the direct interaction of the starting materials, owing to their $\mathrm{pKa}$ differences (BTC; I 3.12, II 3.89,III 4.70 and acetic acid 4.70).

Hence a reactive crystallization strategy was optimized to crystallize the title complex in the aqueous medium at room temperature itself. As described in the experimental section, the zinc source, (zinc acetate) was taken in the aqueous medium. In the next stage, the BTC ligand was first dissolved in methanol, in order to improve the aqueous solubility of the ligand, and was slowly added to the aqueous zinc source, and left for crystallization without any disturbance. It is interesting to note that, this simple modification itself is very efficient to yield the title complex quite readily within 12 hours, but a significant yield of $45 \%$ could be easily obtained by just decanting the mother liquor. The single crystal X-ray diffraction study on the harvested crystalline material, confirms that the structural features of the crystalline material are identical with those of the previous reports [10,11] (Figure 1), and thus the title complex could be readily prepared in the direct crystallization method too, as envisaged. 


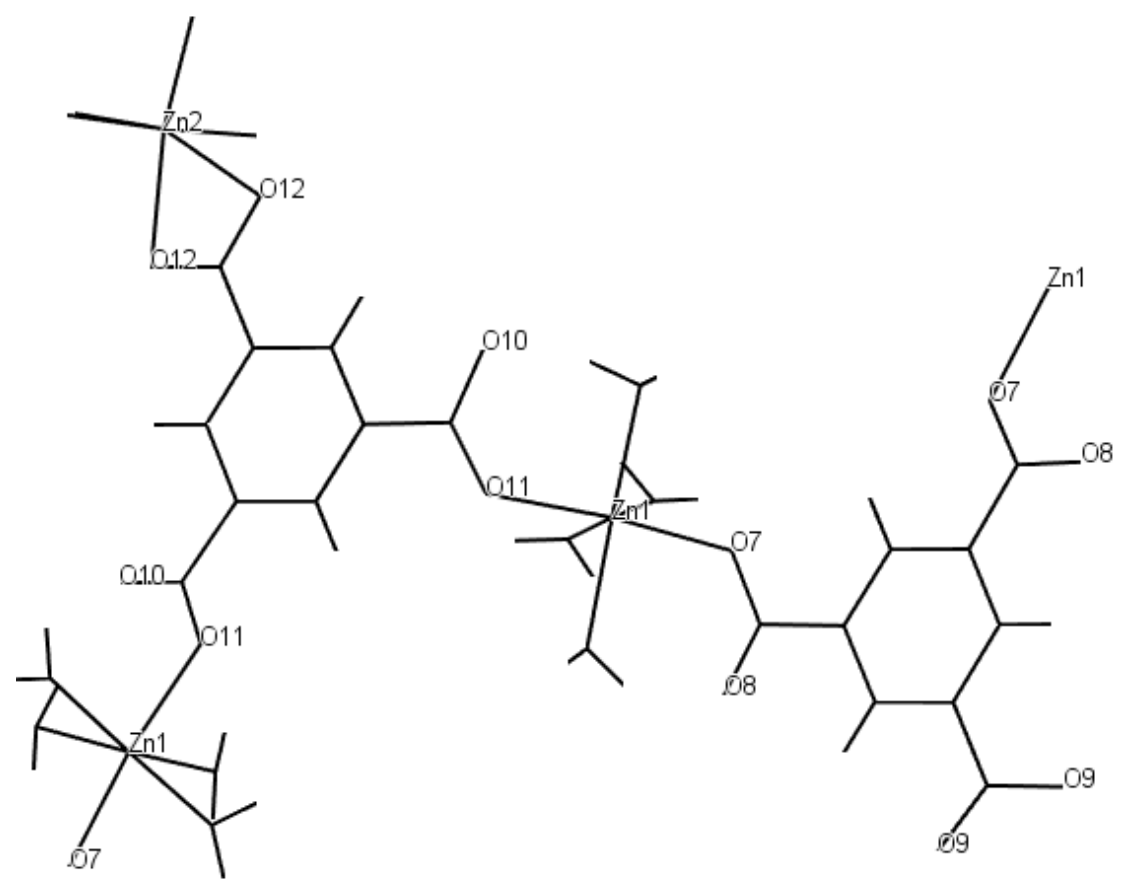

Figure 1. Perspective structural view of the material

It is quite clear from this discussion that the harvesting of the title complex was indeed effortless in this newer method, and the crystallization actually takes place in the aqueous environment itself, under room temperature, without invoking any hydrothermal conditions or other organic bases $[10,11]$. It is also worth mentioning that the present strategy straight away yields the desired complex, without the formation of any uncharacterized precipitates [11]. Hence, this newer route is regarded as an environmentally benign method for the crystallization of $\left[\mathrm{Zn}_{3}(\mathrm{BTC})_{2}\left(\mathrm{H}_{2} \mathrm{O}\right)_{12}\right]_{\mathrm{n}}$ complex for a bulk yield. The scheme 1 describes the optimized synthesis of the title complex in the aqueous medium at room temperature.

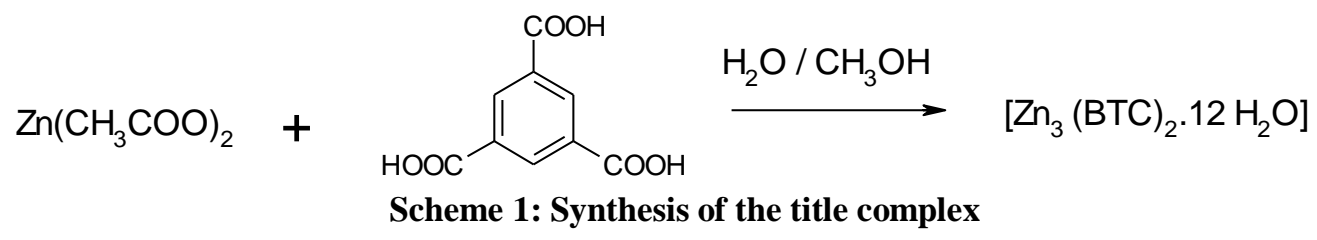

The PXRD profiles, matching to that of the calculated pattern (Figure 2), indicates the phase purity of the products in bulk preparation as well. Elemental analysis further corroborates the phase purities of the prepared sample. The moisture stability and the ability of the structural phase to withstand the dehydration-rehydration shuttles were alsofound to be in line with the results of the Yagi et.al [10]. 


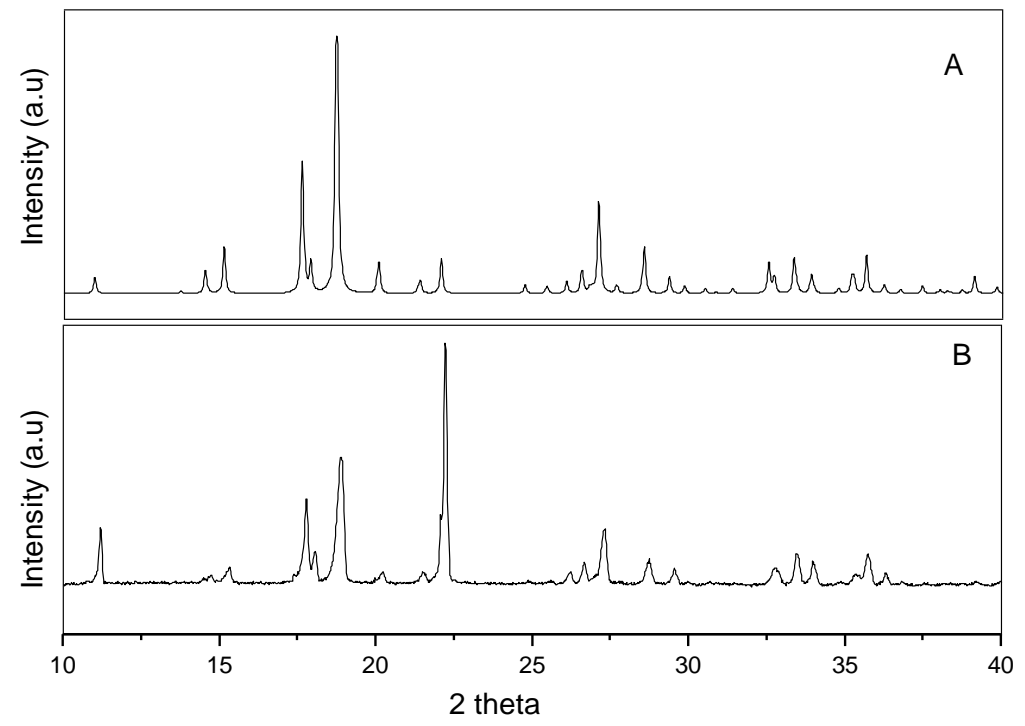

Figure 2 PXRD profiles of the (A) simulated (B) as -synthesized phase of

\subsection{Proton conductivity}

complex

Crystalline samples of complex have been thoroughly ground and pelletized. The sandwiched pellet, after coating with silver paint was used for AC impedance studies, to examine the proton transport characteristics of the material. The AC impedance of the pellet, under inherent condition itself (without purposeful humidification) shows an easily discernible and meaningful Nyquist plot (Figure 3). The inherent conductivity of the sample, though poor, $\left(5.9 \times 10^{-9} \mathrm{~S} \mathrm{~cm}^{-1}\right)$, puts up the characteristic depressed semicircle nature in the Nyquist plot, and is probably attributed to the coordinated water molecules of the system, that are present even before additional humidification. 


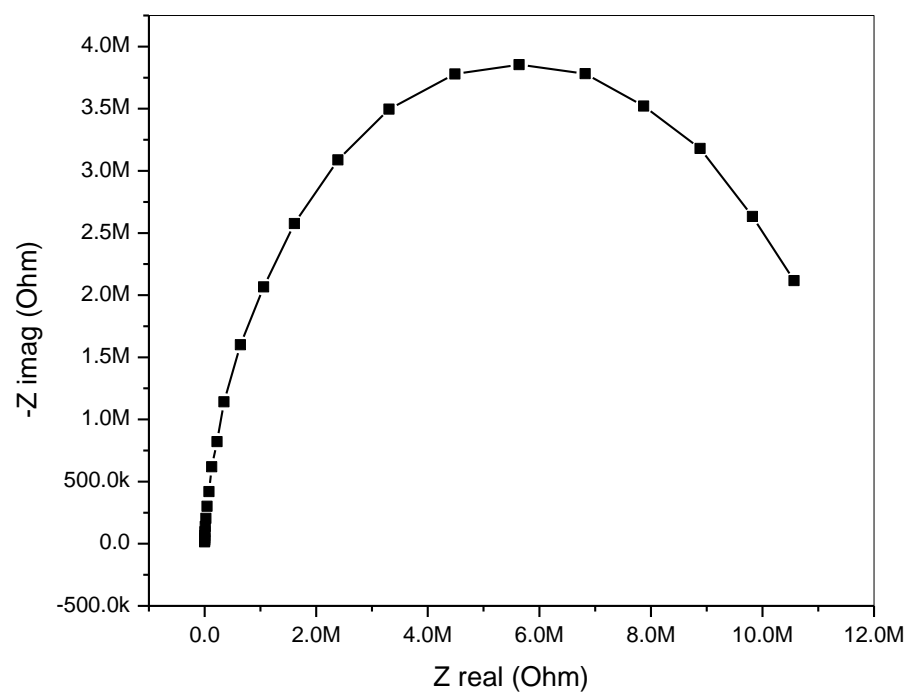

Figure 3. The impedance plot of the complex (at inherent level)

Furthermore, the conductivity of the pelletized sample quickly improves upon exposing to the humidity. Within 2 hours of exposure at $100 \%$ humidity, the Nyquist plot exhibited an appearance of low frequency tail, attributable to the diffusion phenomena also improves. The humidity saturation (100\% RH) is attained in 40 hours duration, during which the Nyquist plot shows a clear semicircle with a prominent low frequency spur, which fits with the equivalent circuit, $R_{1}+\left[C P E / R_{2}\right]+C P E$, where, $R_{1}$ and $R_{2}$ is the lead resistance and bulk resistance of the material respectively, without any grain boundary contribution; CPE represents constant phase elements (Figure 4).

The conductivity of the sample after 44 hours of humidification under $100 \%$ humidity at room temperature, is at the level of $8 \times 10^{-5} \mathrm{Scm}^{-1}$, which is appreciable [13]. 


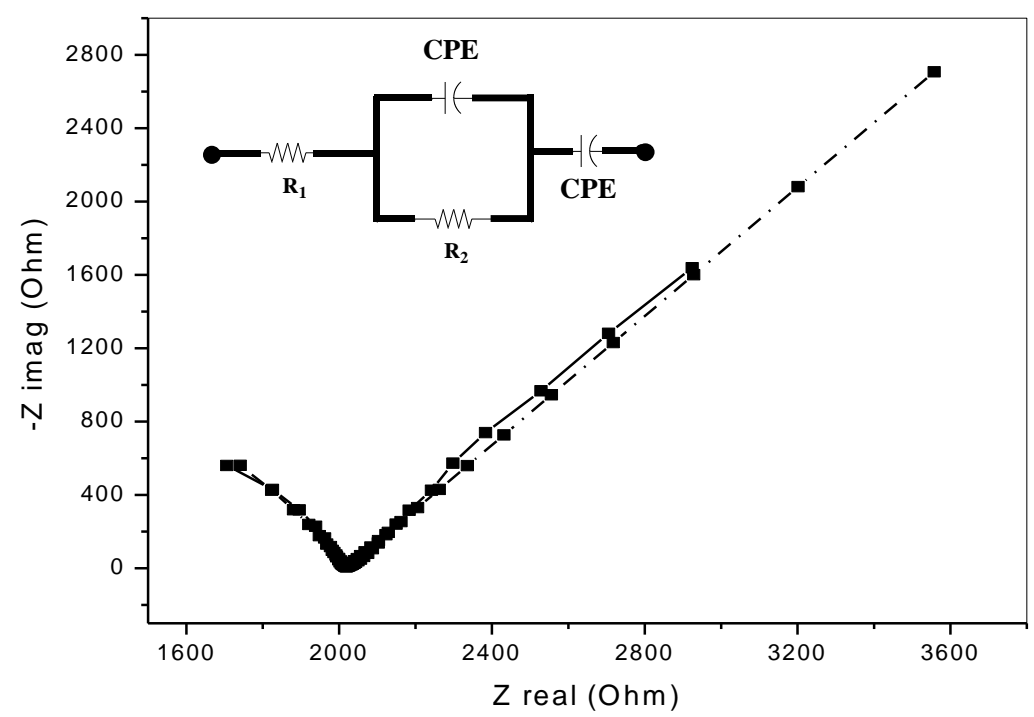

Figure 4. The experimental (- and simulated EIS spectra(- - -) with suitable equivalent circuit of the complex

The reversible dehydration - rehydration shuttle was examined by air-blowing the humidified pellet around $45^{\circ} \mathrm{C}$, during which, the Nyquist plot also exhibited related changes. After 15 minutes of air blowing, the conductivity value of the sample dropped to several orders of magnitude [9] with prominent differences in the Nyquist plot (Figure 5). However, when the pelletized sample was exposed to humidity again, the conductivity of the sample

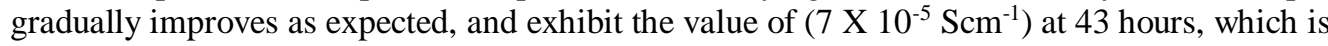
closer to the original value of the system (Figure 6). In addition, it is to be mentioned that the AC impedance characteristics of the pellet, in the presence of pentane vapour, do not exhibit any significant changes with respect to time and is no different from that of the inherent conditions. All these experimental evidences unequivocally proves the need for humidity or water molecules in the formation of a continuous hydrogen bonded conductivity network, thus illustrating the fact that the protons are indeed the charge carrier in the conductivity mechanism. 


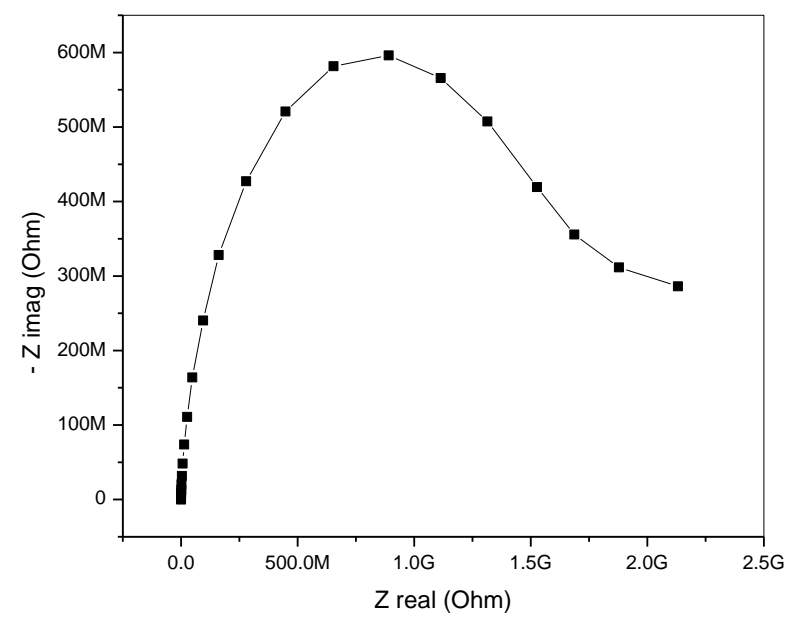

Figure 5. The impedance after dehydration

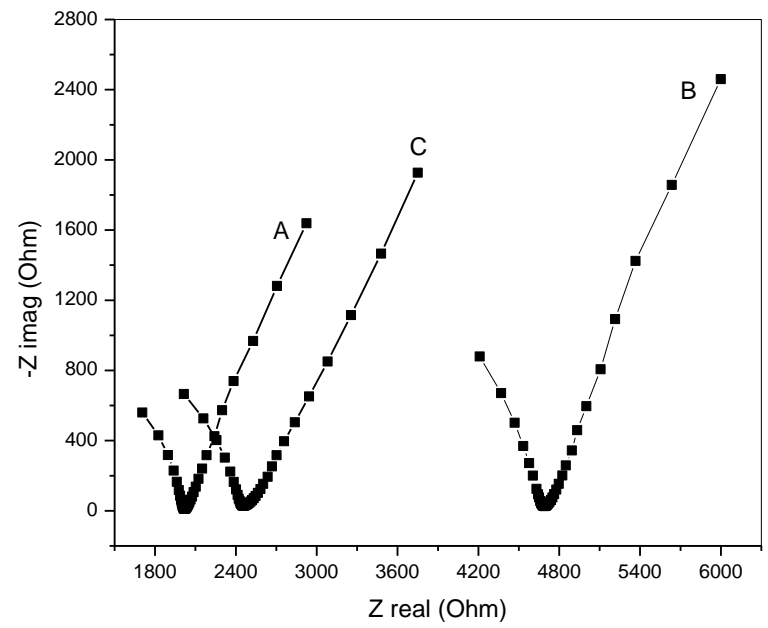

Figure 6. Over lay of impedance profiles; (A) originally prepared sample under $100 \%$ RH (B) Rehydrated for 45 mins and (C) Rehydrated for 43 hours

Temperature dependent AC impedance studies have also been performed to examine the nature of proton transport. As shown in the Figure 7,the conductivity of the sample gradually increases with respect to temperature at $100 \%$ humidity conditions, from which the Arrhenius plot has been obtained. The lower value of energy of activation $\left(E_{a}\right) 0.11 \mathrm{eV}$, calculated from the slope of the Arrhenius plot, proves that only the Grotthuss mechanism is obeyed during the process [ $[1-7]$. 

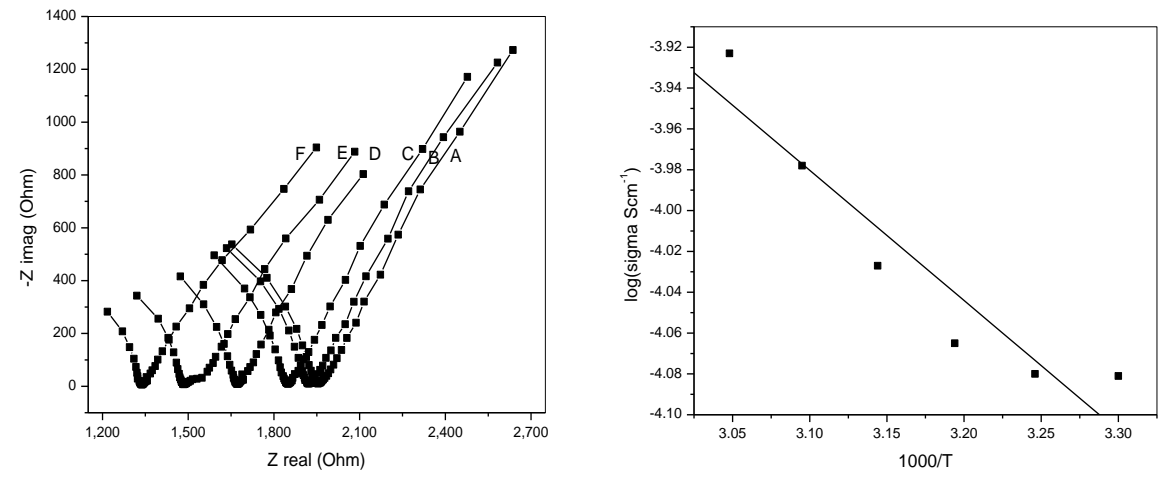

Figure 7.The Arrhenius plot; [Inset (A) $30^{\circ} \mathrm{C},(\mathrm{B}) 35^{\circ} \mathrm{C}$, , (C) $40^{\circ} \mathrm{C}$, (D) $45^{\circ} \mathrm{C}$, (E) 50 ${ }^{\circ} \mathrm{C}$ and $\left.(\mathrm{F}) 55^{\circ} \mathrm{C}\right]$

\section{Conclusion}

This work describes an effortless crystallization strategy for the title complex in the solution phase and is regarded as a quicker method when compared to the hydrothermal or slow evaporation based techniques. Further, this work brings out a newer synthetic strategy to crystallize the material under environmentally friendly manner. The authenticity of the material is confirmed through analytical examinations.The salient structural features of the compound, such Lewis acidic coordinated waters, in combination with an extended hydrogen bonded network, have been realized in the present context as proton electrolytes, and thus the complex is foreseen as a useful proton conducting material. The AC impedance characterizations confirm the hypothesis and prove that the material is indeed a proton conductor. The $\mathrm{E}_{\mathrm{a}}$ studies indicate the Grothussmechanism of proton conduction.

\section{Acknowledgement}

Authors thank the Principal and Management of the PSG College of Technology for the facilities. Authors wish to thank the Heads of the Department of Applied Science, and Chemistry for their encouragement. Authors sincerely thank the SAIF - IIT Madras for single crystal data collection, solving and refining of the structure, and the STIC-Cochin for carrying out the elemental analysis.

\section{References}

[1] Ghasempour, H., Wang, K.Y., Powell, J.A., ZareKarizi, F., Lv, X.L., Morsali, A. and Zhou, H.C., 2021. Metal-organic frameworks based on multicarboxylate linkers. Coordination Chemistry Reviews, 426, p.213542.

[2] Lu, X.F., Fang, Y., Luan, D. and Lou, X.W.D., 2021. Metal-organic frameworks derived functional materials for electrochemical energy storage and conversion: a mini review. Nano Letters, 21(4), p.1555-1565. 
[3] Ryu, U., Jee, S., Rao, P.C., Shin, J., Ko, C., Yoon, M., Park, K.S. and Choi, K.M., 2021. Recent advances in process engineering and upcoming applications of metal-organic frameworks. Coordination Chemistry Reviews, 426, p.213544.

[4] Cai, Y.Y., Yang, Q., Zhu, Z.Y., Sun, Q.H., Zhu, A.M., Zhang, Q.G. and Liu, Q.L., 2019. Achieving efficient proton conduction in a MOF-based proton exchange membrane through an encapsulation strategy. Journal of Membrane Science, 590, p.117277.

[5] Xie, X.X., Yang, Y.C., Dou, B.H., Li, Z.F. and Li, G., 2020. Proton conductive carboxylate-based metal-organic frameworks. Coordination Chemistry Reviews, 403, p.213100.

[6] Afrin, U., Mian, M.R., Otake, K.I., Drout, R.J., Redfern, L.R., Horike, S., Islamoglu, T. and Farha, O.K., 2021. Proton Conductivity via Trapped Water in Phosphonate-Based Metal-Organic Frameworks Synthesized in Aqueous Media. Inorganic chemistry, 60(2), p.1086-1091.

[7] Karimi, M.B., Hooshyari, K., Salarizadeh, P., Beydaghi, H., Ortiz-Martínez, V.M., Ortiz, A., Uribe, I.O. and Mohammadi, F., 2021. A comprehensive review on the proton conductivity of proton exchange membranes (PEMs) under anhydrous conditions: Proton conductivity upper bound. International Journal of Hydrogen Energy, 46(69), p.34413-34437.

[8] Sarango-Ramírez, M.K., Park, J., Kim, J., Yoshida, Y., Lim, D.W. and Kitagawa, H., 2021. Void Space vs. Surface Functionalization for Proton Conduction in Metal-Organic Frameworks. AngewandteChemie International Edition, 60(37), p.20173-20177.

[9] Saravanabharathi, D., Obulichetty, M., Rameshkumar, S. and Kumaravel, M., 2012. Rapid crystallization and proton conductivity of copper (II)-l-tartrate. Synthetic metals, 162(17-18), p.1519-1523.

[10] Yaghi, O.M., Li, H. and Groy, T.L., Journal of the American Chemical Society (1996) 118(38), p.9096-9101.

[11] Krishnamurthy, D. and Murugavel, R., 2003. Reactions of 1, 3, 5-benzenetricarboxylic acid with $\mathrm{Zn}$ (II) ion in the presence of added amines: Isolation and structure determination of zinc coordination polymers with tetrahedral and octahedral zinc centres. Indian Journal of chemistry Section A, 42(9), p.2267-2276.

[12] G.M. Sheldrick, SHELXS97, SHELXL97, Programs for Crystal Structures Solution and Refinement, University of Göttingen, Germany, 1997

[13] Lim, D.W. and Kitagawa, H., 2021. Rational strategies for proton-conductive metal-organic frameworks. Chem. Soc. Rev., 50, p.6349-6368. 